\title{
Is the answer just beneath the surface? And other updates on recent autonomic research
}

\author{
Mitchell G. Miglis ${ }^{1} \cdot$ Srikanth $_{\text {Muppidi }}{ }^{1}$
}

Received: 10 September 2017 / Accepted: 25 September 2017 / Published online: 5 October 2017

(C) Springer-Verlag GmbH Germany 2017

\section{Is the answer just beneath the surface?}

The skin punch biopsy has been around for decades, having been traditionally used by dermatologists to evaluate suspicious skin lesions. More recently, neurologists have been recognizing its value as a diagnostic tool in patients with suspected small fiber neuropathy. In addition, researchers over the past several years have been working on a reliable assay to evaluate skin samples for $\alpha$-synuclein deposition. The use of skin biopsy to evaluate phosphorylated $\alpha$-synuclein (p-syn) has been demonstrated in patients with synucleinopathies such as Parkinson disease (PD) and pure autonomic failure [1] and, more recently, idiopathic REM sleep behavior disorder [2].

Now, in the July 25th issue of Neurology, Donadio and colleagues report on the use of skin biopsy for the diagnosis of dementia with Lewy bodies (DLB), another synucleinopathy [3]. The investigators took punch biopsies from 3 sites in 18 patients with DLB: the paravertebral cervical area, the proximal thigh, and the distal leg. Patients were rigorously selected, and all underwent either cardiac iodine-123 metaiodobenzylguanidine (MIBG) scans or nigrostriatal dopamine transporter ligand [123I] ioflupane (DaT) scans to help confirm the diagnosis. Epidermal tissue was stained with fluorescent antibodies to identify p-syn. Results were compared to 25 healthy controls and 23 patients with presumed non-synucleinopathy dementia, including 13 patients with Alzheimer's disease, 6 patients with frontotemporal

Srikanth Muppidi

muppidis@stanford.edu

1 Stanford Medical Center, Stanford Neurosciences Health Center, 213 Quarry Road, 2nd Floor, Palo Alto, CA 94304, USA dementia, and 4 with vascular dementia. P-syn deposition was present in $100 \%$ of DLB patients and in no controls. There was a proximal-distal gradient observed, with the cervical site showing the greatest concentration of p-syn $(100 \%$ positive). Adrenergic fibers in arterioles, small nerve bundles near arterioles in the deeper layer of the dermis, and the arrector pili muscles were most affected, with deposition also occurring in parasympathetic cholinergic fibers [sweat glands innervated by cholinergic vasoactive intestinal polypeptide (VIP)-positive fibers]. Nine patients had tilt-table testing, which confirmed orthostatic hypotension $(\mathrm{OH})$. One patient had $\mathrm{OH}$ documented on office orthostatic measurements, and one patient was classified as having autonomic impairment based on erectile dysfunction and sweat loss with an absent sympathetic skin response. The remainder of the patients did not have $\mathrm{OH}$ on orthostatic measurements and did not undergo tilt-table testing. Of interest to autonomic practitioners, patients who complained of autonomic symptoms had $100 \%$ positivity in all sites sampled, whereas patients who did not report autonomic symptoms had $100 \%$ positivity only at the cervical site.

The rate of positivity was quite surprising and much higher than reported in patients with idiopathic PD. According to the authors, "a remarkable aspect of the current data is the higher amount of p-syn in skin samples analyzed in the patients with DLB (75\%) than previously reported in PD (49\%) using the same methods. This finding suggests a more widespread involvement of autonomic nerves in DLB and explains the higher frequency of $\mathrm{OH}$ in DLB than PD." Interestingly, this pattern of widespread p-syn deposition is similar to what was seen in skin biopsy samples of patients with pure autonomic failure.

There are some limitations to this study, including the small sample size and the younger age of the patients (mean age of symptom onset was 59 years), considerably younger 
than most patients with dementia. The patients were rigorously selected and may not represent the more variable population of a typical neurology clinic. All this limits generalizability, and we await future studies to help validate the sensitivity and specificity of skin biopsy for p-syn in the synucleinopathies. Nonetheless, this study and others add to the growing body of literature that supports skin biopsy as a valuable diagnostic tool, and we hope that it signals a future of less-invasive testing for patients. It also reminds us that sometimes, despite an exhaustive search, the answer may be lurking just beneath the surface.

\section{Ivabradine and postural tachycardia syndrome: the evolving armamentarium}

From its early description in the late nineteenth century to its rapid growth in recent years as one of the most frequently diagnosed autonomic syndromes, postural tachycardia syndrome (POTS) has proven a challenge for patients and physicians alike. While there is no standard of care for treatment, many medications are prescribed off-label for symptomatic relief, with variable and inconsistent results. What works for one patient may cause significant side effects in another, and many patients remain desperate for a cure. Physicians are left with only their prior experience and limited evidence to guide them, while many patients remain quite disabled by their symptoms. Thus, when a new medication promises positive results, the POTS world—patients, families, patientadvocacy groups, and physicians alike-take notice.

Ivabradine appears to be the newest such medicine. Its mechanism of action relies on funny channel blockade at the sinoatrial node, resulting in a reduction in heart rate without a decrement in blood pressure. Funny channels underlie the cardiac "pacemaker" current, an inward current that flows though intermembrane cardiac proteins with mixed sodium and potassium permeability and play a vital but still incompletely understood role in cardiac rate generation. In the August 28th issue of Pacing Clinical Electrophysiology, Ruzieh and colleagues report on their experience with ivabradine in patients with POTS [4]. Forty-nine patients with POTS received ivabradine in their clinic. All patients were initially prescribed the starting dose of $2.5 \mathrm{mg}$ twice daily and titrated to a maximum dose of $10 \mathrm{mg}$ twice daily. Thirty-eight of these patients (76\%) reported symptomatic improvement. The authors note that those patients who experienced symptomatic improvement had a significantly lower heart rate during sitting $(78.1 \pm 10.7$ vs. $72.5 \pm 7.6)$ and standing (107.4 \pm 14.1 vs. $95.1 \pm 13.7)$, though the mean change in heart rate on standing was not noted. The average number of medications trialed before ivabradine was $3 \pm 1.5$, which recapitulates our experience in managing
POTS patients. The medication was very well tolerated, with no discontinuations due to adverse events.

There are, however, many limitations that lessen the enthusiasm for this work. It is a retrospective chart review without well-defined outcomes and no control group. There is no mention of tilt-table testing or other cardiovascular autonomic parameters in these patients, and no mention of how the work-up of these patients was performed or the diagnosis of POTS was obtained. The mean age of the patients was $35.1 \pm 10.5$, which is slightly older than the typical POTS demographic. Although the authors state that $42.9 \%$ of their patients had "hypermobility" and $2 \%$ were "hyperadrenergic," it is not clarified how these diagnoses were determined. One patient had diabetes and another had atrial fibrillation, which confounds the sample.

Nevertheless, to our knowledge, this is the largest cohort of POTS patients in whom the use of ivabradine has been reported, and the results appear to be promising. In a prior mechanistic study, ivabradine was compared to metoprolol and placebo in double-blind, crossover fashion in a population of 19 healthy men without cardiac or autonomic disease [5]. All subjects underwent tilt-table testing with continuous blood pressure and heart rate monitoring, and all subjects were given reboxetine, a norepinephrine transporter inhibitor, to induce excessive cardiac sympathetic stimulation. The tilt protocol in this study was more gradual than typical in standard practice: the investigators increased the tilt angle by $15^{\circ}$ every 2 min up to a maximum angle of $60^{\circ}$, at which subjects remained for $20 \mathrm{~min}$. Lower-body negative pressure was administered during tilt in all subjects for added orthostatic stress.

Blood pressure was better maintained with ivabradine, and cardiac stroke volume was reduced with metoprolol but not with ivabradine. Unlike the results reported by Ruzieh et al, ivabradine in this study did not reduce heart rate at rest (as compared to metoprolol, which did), while during orthostatic stress and norepinephrine transporter blockade, there was a significant reduction in heart rate (although to a lesser extent than metoprolol). This suggests that ivabradine may be more effective during states of increased cardiac sympathetic drive, one proposed etiology of POTS.

Because ivabradine is not specifically Food and Drug Administration (FDA)-approved to treat POTS, insurance authorization remains a major challenge in the United States, and with an average cost of $\$ 400$ per month, expense is a significant barrier. Randomized placebo-controlled trials are required to provide solid evidence of efficacy, reduce drug costs, and lead to standards of care for the treatment of this difficult and incompletely understood condition. Despite some preconceptions about POTS, we must not forget that patients with this condition comprise a sizeable portion of disability in the young workforce, resulting in a significant 
loss of productivity on a national scale. Effective treatments cannot come sooner.

\section{Autonomic dysfunction and survival in the $\alpha$-synucleinopathies}

Autonomic dysfunction is a common feature of the $\alpha$-synucleinopathies, and tends to portend a worse prognosis. Patients with $\mathrm{PD}$ and $\mathrm{OH}$ have a greater mortality risk than patients with PD without $\mathrm{OH}$ [6]. However, it is unclear if autonomic dysfunction in PD is associated with more rapid progression of disease and worse overall survival. In a recent article in JAMA Neurology, De Pablo-Fernandez and colleagues report on the association of autonomic dysfunction, disease progression and survival in autopsy-proven cases of PD [7]. Investigators performed a retrospective review of clinical data from 100 consecutive patients who had an autopsy-confirmed diagnosis of PD from 2006 to 2016. These patients were seen by either a neurologist or a geriatrician for symptom management. Autonomic dysfunction was defined as autonomic failure diagnosed by autonomic testing or at least two symptoms/signs of autonomic impairment: urinary symptoms, constipation, upper gastrointestinal dysfunction, $\mathrm{OH}$, sweating abnormalities, or erectile dysfunction. Time to development of six disease milestones (predefined as frequent falls, cognitive impairment, unintelligible speech, severe dysphagia, dependence on wheelchair for mobility, and placement in residential or nursing home) along with time to development of autonomic dysfunction was analyzed.

Among the cohort of 100 patients, 60 were men. The mean age at time of diagnosis was 63.9 years, and the mean disease duration was 14.6 years. Autonomic dysfunction was noted in 85 patients, with a mean time to onset of autonomic dysfunction of 6.7 years after PD diagnosis. Autonomic dysfunction was associated with the following variables: older age at the time of diagnosis in men, poor response to levodopa, and postural or gait instability. Interestingly, pathological changes on brain autopsy did not correlate with the degree of autonomic symptomatology. Patients with early autonomic dysfunction had an increased risk of reaching the various PD milestones [hazard ratio (HR) $0.86, p<0.001$ ] and had a shortened survival (HR 0.92, $p<0.001$ ). Based on this analysis, the authors propose that patients with PD who have autonomic dysfunction tend to have a shorter lifespan and reach PD diseases milestones at a faster rate. While this is one of the larger studies to use autopsy-proven
PD to assess the effect of autonomic failure on disease progression, it is mainly limited by its retrospective nature. In addition, patients were managed by a range of physicians and there could be variability in clinical practice. Also, common autonomic symptoms do not always reflect the degree of autonomic failure. Further, it is unclear if the physicians used validated clinical questionnaires to identify autonomic dysfunction. Finally, the study did not mention if the treatments for $\mathrm{OH}$ were uniform amongst all patients and, if so, what treatments were used.

Despite these limitations, this study emphasizes the importance of screening for autonomic dysfunction in patients with PD, either by clinical symptom review or by autonomic testing, as early recognition and appropriate treatment could reduce symptom burden and extend survival in PD.

\section{Compliance with ethical standards}

Conflict of interest The author declare that they have no competing interest.

Funding support None.

\section{References}

1. Donadio V, Incensi A, Piccinini C et al (2016) Skin nerve misfolded $\alpha$-synuclein in pure autonomic failure and Parkinson disease. Ann Neurol 79:306-316. doi:10.1002/ana.24567

2. Doppler K, Jentschke H-M, Schulmeyer L et al (2017) Dermal phospho-alpha-synuclein deposits confirm REM sleep behaviour disorder as prodromal Parkinson's disease. Acta Neuropathol 133:535-545. doi:10.1007/s00401-017-1684-z

3. Donadio V, Incensi A, Rizzo G et al (2017) A new potential biomarker for dementia with Lewy bodies: skin nerve $\alpha$-synuclein deposits. Neurology 89:318-326. doi:10.1212/ WNL.0000000000004146

4. Ruzieh M, Sirianni N, Ammari Z et al (2017) Ivabradine in the treatment of postural tachycardia syndrome (POTS), a single center experience. Pacing Clin Electrophysiol. doi:10.1111/ pace. 13182

5. Schroeder C, Heusser K, Zoerner AA et al (2014) Pacemaker current inhibition in experimental human cardiac sympathetic activation: a double-blind, randomized, crossover study. Clin Pharmacol Ther 95:601-607. doi:10.1038/clpt.2014.25

6. Goldstein DS, Holmes C, Sharabi Y, Wu T (2015) Survival in synucleinopathies: a prospective cohort study. Neurology 85:1554-1561. doi:10.1212/WNL.0000000000002086

7. De Pablo-Fernandez E, Tur C, Revesz T et al (2017) Association of autonomic dysfunction with disease progression and survival in parkinson disease. JAMA Neurol 74:970. doi:10.1001/ jamaneurol.2017.1125 